Fazit für die Praxis:

Bedingt durch die demografische Entwicklung, aber auch durch deutlich bessere Therapieoptionen, nimmt die Zahl der an Krebs erkrankten Patienten weltweit kontinuierlich zu. Gleichzeitig können immer mehr Patienten von der Erkrankung geheilt werden, sodass die Zahl der "longterm survivor" kontinuierlich steigt. Tumorkrankheiten präsentieren sich damit zunehmend als chronische Krankheit. Diese erfreuliche Entwicklung rückt das Problem der Langzeitfolgen von Krebserkrankung und -therapie in den Fokus des therapeutischen Interesses. Häufig werden dabei aber die finanziellen Konsequenzen für Patienten und deren Angehörigen übersehen.

Die hier dargestellten Zahlen belegen, wie ungeheuer wichtig eine frühzeitige Aufklärung von Krebspatienten über die gesetzlichen Regelungen und deren Konsequenzen ist. Denn es besteht die Möglichkeit, dass Patienten an den Folgen der Erkrankung und/oder Therapie verarmen. Das Verarmungsrisiko betrifft neben den finanziellen Fragen, die häufig mit Problemen in der Familie und im sozialen Umfeld einhergehen, auch die Lebensqualität und möglicherweise nicht zuletzt die Prognose unserer Patienten. Aus diesem Grunde sollte, wenn möglich, schon während der Tumortherapie an die Bewältigung dieser „Nebenwirkung“ der Krebserkrankung bzw. deren Therapie gedacht werden. Insbesondere bei $\mathrm{Pa}$ tienten im Alter zwischen 40 und 60 Jahren die eine geringere Schulbildung (kein Abitur) besitzen und einer mittelschweren bis schweren Tätigkeit nachgehen, sollte der Themenkomplex schon mit dem Auslaufen der Lohnfortzahlung angesprochen werden. Hilfestellungen können über die Sozialdienste der Kliniken, Selbsthilfegruppen, den Sozialverband $\mathrm{VdK}$, die Krebsberatungsstellen, aber insbesondere auch in der onkologischen $\mathrm{Re}$ habilitation geleistet werden.

\section{Literaturverzeichnis}

1. Krebs in Deutschland 2011/2012. 10. Ausgabe. Robert Koch-Institut und die Gesellschaft der epidemiologischen Krebsregister in Deutschland e.V. (Hrsg). Berlin, 2015.

2. DeSantis $C E$ et al. Cancer treatment and survivorship statistics, 2014. CA Cancer J Clin. 2014;64(4):252-71.

3. World estimated cancer prevalence, adult population: both sexes. http://globocan. iarc.fr/summary_table_pop_prev.asp?selec tion $=225900 \&$ title $=$ World $\&$ sex $=0$ \&window $=1 \&$ sort $=0 \&$ submit $=\%$ A0Execute $\%$ A0 (Accessed on February 13, 2013).

4. Epidemiologist, Surveillance and Health Services Research, American Cancer Society, Atlanta, GA.GLOBOCAN 2008 Update http:// www.iarc.fr/en/media-centre/iarcnews/2011/globocan2008-prev.php (Accessed on July 16, 2012).

5. Rick $O$ et al. Reintegrating cancer patients into the workplace. Dtsch Arztebl Int. 2012;109(42):702-8.

6. de Boer AG et al. Cancer survivors and unemployment: a meta-analysis and meta-regression. JAMA. 2009;301(7):753-62.

7. Dowling EC et al. Lost productivity and burden of illness in cancer survivors with and without other chronic conditions. Cancer. 2013;119(18):3393-401.

8. Mehnert A. Employment and work-related issues in cancer survivors. Crit Rev Oncol Hematol. 2011;77(2):109-30.

9. Luengo-Fernandez $\mathrm{R}$ et al. Economic burden of cancer across the European Union: a population-based cost analysis. Lancet Oncol. 2013;14(12):1165-74.

10. Delgado-Guay MO et al. Frequency, intensity, and correlates of financial distress (FD) among advanced cancer patients (AdCa). J Clin Oncol. 2014;32(5s, Suppl):Abstr 9635.
11. Strik H. Impact and effects of sleep disturbances in younger cancer patients. Oncol Res Treat 2015;38(suppl 5):v90.

12. Daten des BKK Bundesverbandes, Die Zeit 13.08.2012

13. Seifart $U$ et al. Armut durch Krebs - Wenn Menschen als Folge der Erkrankung in soziale Notlagen geraten. Hess Ärztebl. 2016;77(3):145-7.

14. de Boer AG et al. Interventions to enhance return-to-work for cancer patients. Cochrane Database Syst Rev. 2015;9:CD007569.

15. American Cancer Society. Cancer Treatment and Survivorship Facts \& Figures 2012-2013. http://www.cancer.org/research/cancerfactsstatistics/survivor-facts-figures.

\section{PD Dr. med. Ulf Seifart}

Klinik Sonnenblick, Akademisches Lehrkrankenhaus der Universität Marburg Baldinger Str. 1-6, 35043 Marburg, ulf.seifart@drv-hessen.de

Für die Arbeitsgemeinschaft Supportive Maßnahmen in der Onkologie, Rehabilitation und Sozialmedizin der Deutschen Krebsgesellschaft (ASORS). ASORS im Internet: www.asors.de

\title{
Ärzte und Ärztinnen für eine (Online-) Befragung gesucht
}

Hinter dem Momentum-Projekt Heidelberg verbirgt sich eine wissenschaftliche Studie zum Umgang mit einer Krebserkrankung. Befragt werden sollen Ärzte und Ärztinnen, vor allem aus den Bereichen Allgemeinmedizin, Gynäkologie, Urologie, Gastroenterologie, Chirurgie und Strahlentherapie, die in ihrem Behandlungsalltag regelmäßig Kontakt zu Patienten bzw. Patientinnen mit Brust-, Darm- oder Prostatakrebs haben. Untersucht wird die ärztliche Sicht auf Strategien und Maßnahmen, die an Krebs erkrankte Personen zusätzlich zu der medizinischen Behandlung anwenden können. Das sind etwa ernährungsbezogene Strategien oder körperliche Aktivität. Gefragt wird z.B. "Was halten Sie für sinnvoll?" "Welche Empfehlungen zusätzlich zur medizinischen Behandlung geben Sie und wovon raten Sie eher ab?"

Die Ergebnisse der Studie sollen dazu beitragen, die ärztliche Beratungssituation für alle Beteiligten zu verbessern. Außerdem werden auf Basis der Resultate Angebote und Informationsmaterialien für Patientinnen und Patienten entwickelt.

Beteiligt am Momentum-Projekt sind die Universität Heidelberg (Prof. Dr. Monika Sieverding), das Deutsche Krebsforschungszentrum (Prof. Dr. Karen Steindorf), das Universitätsklinikum Heidelberg (Dr. Joachim Wiskemann) und das Nationale Centrum für Tumorerkrankungen in Heidelberg. Das Projekt wird von der Deutschen Krebshilfe gefördert.

Noch bis Mitte Juli können Interessierte an der Befragung über die Internetseite www.momentum-projekt.de teilnehmen. Hierfür sollten Sie etwa 15-20 Minuten Zeit einplanen. Alternativ können Sie den Fragebogen auch in einer Papierversion anfordern (mit frankiertem Rückumschlag). Schreiben Sie hierfür eine Email an: momentum@dkfz.de

Teilnehmerinnen und Teilnehmer erhalten eine Aufwandsentschädigung in Höhe von 25 Euro. 